\title{
El uso continuo de la amiodarona es superior al uso episódico para la prevención de la fibrilación auricular
}

\author{
Continuous use is superior to episodic use for the prevention of atrial fibrillation
}

Ahmed S y col. JAMA 2008;300(15):1784-92

\section{Objetivo}

Comparar la incidencia de eventos mayores en paciente que reciben tratamiento con amiodarona en forma continúa vs. los que la reciben en forma episódica para prevenir la fibrilación auricular (FA).

\section{Diseño, lugar y pacientes}

Ensayo clínico aleatorizado con un seguimiento promedio de 2,1 años, que tomó lugar en siete centros de los países bajos. Fueron incluidos 209 pacientes ambulatorios con FA recurrente sintomática persistente o aleteo auricular con una duración menor a un año y una frecuencia cardíaca mayor a 75 latidos por minuto. Fueron excluidos los pacientes con más de tres episodios de FA durante los últimos tres años, uso de amiodarona durante los tres meses precedentes, historia de recaída en el contexto de estar con niveles adecuados de amiodarona, clase funcional III o IV, contraindicaciones para el uso de amiodarona, historia de disfunción tiroidea, tratamiento concomitante con antiarritmicos clase I o III, enfermedad del nodo sinusal, bloqueo aurículoventricular de segundo o tercer grado o marcapasos.

\section{Intervención y medición de resultados principales}

Luego de una carga con amiodarona de $600 \mathrm{mg} /$ día durante cuatro semanas, se les realizó cardioversión eléctrica a los que no habían revertido y a los que quedaron en ritmo sinusal se los aleatorizó a: 1) tratamiento continuo (200 mg/día); 2) suspensión luego de cuatro semanas de la aleatorización y tratamiento episódico de la recurrencia de FA con amiodarona durante un mes pre y post-cardioversión. El punto final primario compuesto fue eventos mayores asociados a cardiopatía subyacente y a amiodarona; y los secundarios, mortalidad global y hospitalización cardiovascular.

\section{Resultados principales}

No hubo diferencia significativa en el punto final primario pero 51 pacientes (48\%) que recibieron tratamiento episódico y $64(62 \%)$ que lo recibieron continuo conservaron ritmo sinusal $(p=0,05)$ durante el seguimiento. Hubo $80 \%$ (85) de recurrencia de FA entre quienes recibieron tratamiento episódico y $54 \%$ (56) en los que lo recibieron en forma continua. $(p<0,001)$. La mortalidad global y la incidencia de hospitalización cardiovascular fue menor en los que recibieron tratamiento continuo (tabla 1).

Tabla 1: eficacia del tratamiento contínuo vs. episódico de la fibrilación auricular con amiodarona.

\begin{tabular}{c|c|c|c|c|c} 
Resultado & $\begin{array}{c}\text { Amiodarona } \\
\text { continua }\end{array}$ & $\begin{array}{c}\text { Amiodarona } \\
\text { episódica }\end{array}$ & $\begin{array}{c}\text { RRR } \\
\text { (IC95\%) }\end{array}$ & $\begin{array}{c}\text { NNT } \\
\text { (IC95\%) }\end{array}$ & P \\
\hline Secundario* & $0,9 \%$ & $0,5 \%$ & $0,4 \%$ & 0.56 & 0,02 \\
& & & $(0,17$ a 0,64$)$ & $(0,39$ a 0,82$)$ & \\
\hline
\end{tabular}

*Se consideró sólo el primer evento ocurrido durante el seguimiento promedio de 2,1 años

\section{Conclusiones}

Hubo una mayor incidencia de recurrencia de FA, de mortalidad global y de hospitalización cardiovascular en quienes recibieron el tratamiento en forma episódica

\section{Comentario}

La FA es la arritmia más frecuente y se asocia con un aumento del riesgo de accidentes embólicos, insuficiencia cardíaca congestiva y mortalidad total. Hace algunos años se publicaron trabajos que mostraron que no existen diferencias clínicas en el manejo de la fibrilación auricular en cuanto al control del ritmo o el control de la frecuencia cardiaca'. Esto fue probablemente debido al mal desempeño de los tratamientos farmacológicos para controlar el ritmo, ya que un reporte post hoc del estudio AFFIRM demostró una mejor sobrevida de los pacientes que permanecían en ritmo sinusal vs. los que continuaban con $\mathrm{FA}^{2}$.

Aunque la terapia antiarrítmica es considerada la primera línea terapéutica en pacientes con FA, la recurrencia es alta, llegando al 44-67\% dentro del primer año post cardioversión eléctrica. La amiodarona continúa siendo la droga más efectiva. Algunos trabajos reportan un mantenimiento del ritmo sinusal de 45 a $70 \%$ en seguimientos de 12 a 54 meses, pero con una importante tasa de efectos tóxicos. Los efectos adversos que resultan en la necesidad de interrumpir el tratamiento tienen una incidencia de 13\% a $18 \%$ después del año. Si bien no hay estudios que avalen el uso de bajas dosis de amiodarona, su prescripción es una práctica común en pacientes añosos con bajo peso corporal ${ }^{3}$. Las limitaciones de este trabajo son su escaso número de pacientes, su corto seguimiento; y haber comparado pacientes con FA y con aleteo auricular, arritmias con diferente respuesta a la terapia.

\section{Conclusiones del comentador}

La posibilidad de disminuir los efectos adversos limitando el tiempo del tratamiento resultaba interesante. Sin embargo, no disminuyeron los efectos tóxicos, sino que hubo mayor recurrencia de FA, hospitalizaciones cardiovasculares y mortalidad global. Vale mencionar que en los últimos años ocurrió un importante avance en el campo de la ablación transcateter de esta arritmia lo que ha resultado en bajas tasas de complicaciones y un mejor pronóstico para estos pacientes.

Gustavo Maid [ Sección de Arritmias, Servicio de Cardiología. Hospital Italiano de Buenos Aires gustavo.maid@hiba.org.ar ]

Maid G. El uso continuo de la amiodarona es superior al uso episódico para la prevención de la fibrilación auricular. Evid. actual. práct. ambul; 13(4): 129, Oct-Dic 2010. Ahmed S y col. CONVERT Investigators. Continuous vs episodic prophylactic treatment with amiodarone for the prevention of atrial fibrillation: a randomized trial. JAMA. 2008;300(15):1784-92. PMID: 18854540.

\section{Referencias}

1. Bounhoure J y col. Arrhythmia-induced dilated cardiomyopathies. Bull Acad Natl Med 2006;190(6):1225-35

2. Guglin M y col. Sinus rhythm is associated with fewer heart failure symptoms: Insights from the AFFIRM trial. Heart Rhythm 2010 January 6.

3. Zimetbaum P y col. Amiodarone for atrial fibrillation. N Engl J Med 2007;356(9):935-41. 